\title{
Practise-oriented consideration of the dynamic fast fault current of power park modules in grid protection analysis
}

\author{
Manuel Jäkel ${ }^{\circledR}$, Hendrik Vennegeerts ${ }^{1}$, Albert Moser ${ }^{1}$, Felix Glinka ${ }^{2}$, \\ Armin Schnettler ${ }^{2}$ \\ ${ }^{1}$ FGH e.V., Germany \\ ${ }^{2}$ IFHT, RWTH Aachen University, Germany \\ $凶$ E-mail: manuel.jaekel@fgh-ma.de
}

\begin{abstract}
Protection design in distribution grids is usually undertaken by simple to handle practice-oriented steady-state calculation methods. For the calculation of the minimal short-circuit current models and methods are described in IEC 60909. In the revised version from 2016 constant current sources for modelling of reactive current infeed of converterbased generators due to mandatory grid support in Germany are considered, though the infeed is voltage dependent. Moreover, the dynamic settling process of the reactive current infeed can affect the functionality of the protection, which is not taken into account in steady-state calculations. This study outlines a possibility to estimate the influence of these new dynamic effects on grid protection behaviour in steady-state calculation methods. Different types of decentralised generation (DG) units as well as other influences, e.g. grid topologies, distribution of DG or protection concepts are taken into account. The authors will show that suitable steady-state calculations are sufficient for practical protection analysis. The risk of undesirable reactions of the grid protection can be minimised by the use of the derived general recommendations.
\end{abstract}

\section{Introduction}

The increase of decentralised generation (DG) in low-voltage and medium-voltage (MV) grids and its participation in fault situation system services (e.g. dynamic grid support by fast fault reactive currents $I_{\mathrm{q}}$ ) lead to new challenges in grid protection analysis. However, easy to use calculation methods with models that can be parameterised by information provided by the plant manufacturer and operator during the standardised connection agreement are needed for practical distribution system planning and parameterisation purposes. Therefore, standardised and well established methods for short-circuit current determination are defined in IEC 60909 [1]. The requirements for DG in fault situations defined by the network operator and the realisation by the manufacturer within this defined framework are not entirely considered in these calculation methods. This procedure may therefore lead to results, which are not precise enough under specific conditions for protection system design and parameterisation. For example, $I_{\mathrm{q}}(U)$ dependency of DG with full size converters is neglected.

\section{Protection design in practice}

\subsection{Present protection concept in MV grids}

In German MV distribution grids the definite-time overcurrent protection (OCP) is most widely used. It is usually designed as selective main protection (MP) and backup protection (BP), as shown in Fig. 1.

The fault case in feeder 1 in the image above is recognised by OCP1 as MP and by OCP0 as BP. OCP2 forms the MP for feeder 2 and should not be stimulated in the shown fault case.

In grids without DG and resonant-earthed-neutral system, a correct protection behaviour is achievable by regarding a two-phased fault at the end of the feeder, resulting in the minimum fault current. The complete short-circuit current has its origin in the overlaying grid (HV). OCP0 and OCP1 are recognising the same current value. Here OCP2 is not stimulated.

A graduation between MP and BP can be configured using a longer delay time for OCP0. Basically in relay charts an overlap is prevented, as shown in Fig. 2.

Present guidelines give recommendations for proper delay times [2]. $100 \mathrm{~ms}$ are recommended for the quick time level. In addition, a relay separation of about $300 \mathrm{~ms}$ should be used.

\subsection{Calculation of the corresponding values}

As mentioned above, steady-state calculation methods for the minimal short-circuit current are used in practice, corresponding to standard IEC 60909. Normally dynamic simulations are only applied in specific cases due to their complex parameterisation and long calculation times, particularly for large systems. In simplified approaches uncertainties are covered to certain extend using adjustment factors, defined in the standard. However, the standard only aims at sufficiently correct short-circuit current values at the fault location. Therefore a so called 'pickup reliability factor' $\left(f_{\mathrm{AK}}\right)$ is used in protection parameterisation considering all additional uncertainties. The maximum setting of the protection is given as

$$
I_{\mathrm{AK}}=\frac{I_{k, \min }}{f_{\mathrm{AK}}}
$$

For the MP the usage of a factor of $f_{\mathrm{AK}, \mathrm{MP}}=1.5$ and for the BP of $f_{\mathrm{AK}, \mathrm{BP}}=1.3$ is a common practice [2].

For the lower parameterisation limit a so-called 'pickup security factor' $\left(f_{\mathrm{AL}}\right)$ is used to consider the equivalent calculation of $I_{\mathrm{AL}}$ regarding the maximum operation current $I_{\mathrm{op} \text {,max }}$ :

$$
I_{\mathrm{AL}}=I_{\mathrm{op}, \max } \cdot f_{\mathrm{AL}}
$$




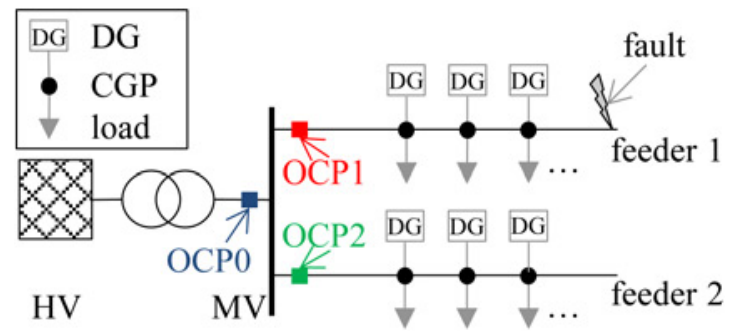

Fig. 1 Illustrative MV distribution grid containing two feeders with MP and $B P$

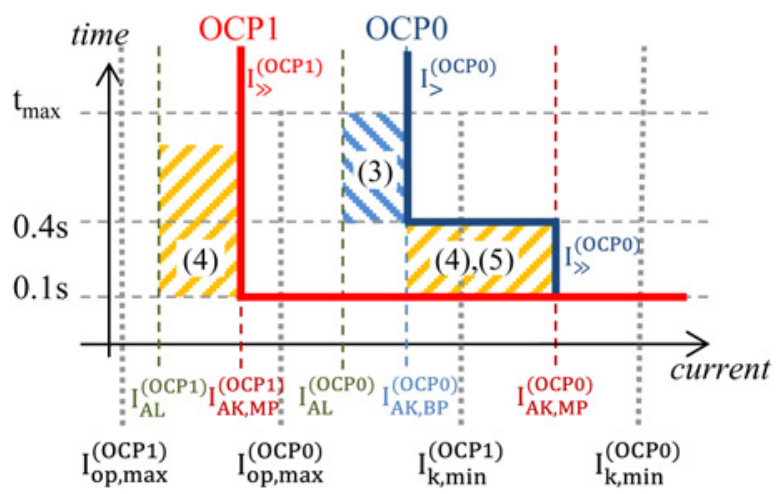

Fig. 2 Simplified protection scaling of OCP0 and OCP1 from Fig. 1, using (3) $-(5)$

Thus, the pickup values for main $\left(I_{?}\right)$ and backup $\left(I_{>}\right)$protection is limited to

$$
\begin{gathered}
I_{\mathrm{AL}} \leq I_{>} \leq I_{\mathrm{AK}, \mathrm{BP}} \\
I_{\mathrm{AL}} \leq I_{\gg} \leq I_{\mathrm{AK}, \mathrm{MP}} \\
I_{>} \leq I_{\gg}
\end{gathered}
$$

In the consequence the shown protection scaling shown in Fig. 2 ensures a selective protective behaviour. Here a fault clearance proceeds after $0.4 \mathrm{~s}$. Provided the MP behaves correctly, the BP's protective pickup will fall back, if the relevant current value falls below the device specific drop-off value $\left(I_{\mathrm{do}}\right)$, mostly around $90 \%$ of the configured $I_{>}$and $I_{\gg}$.

\section{Challenges of specific DG infeed in fault cases}

In Germany DG are required to remain connected during a grid fault according to the standardised connection agreement, the so called low-voltage ride through [3].

The current infeed depends on

- the voltage level,

- specifications of the network operator,

- DG type and DG manufacturer.

In MV grids a so called dynamic grid support by fast reactive fault currents is additionally required for DG plants, e.g. in MV directives $[4,5]$. Here, DGs include power station units with full sized inverters (PF) and doubly-fed induction generators (DFIGs).

\subsection{Requirements on a dynamic grid support by fast fault currents}

3.1.1 Requirements on the reactive current infeed of $D G$ : The specific current infeed of the DG in a fault situation depends on the voltage drop at the grid connection point (GCP).

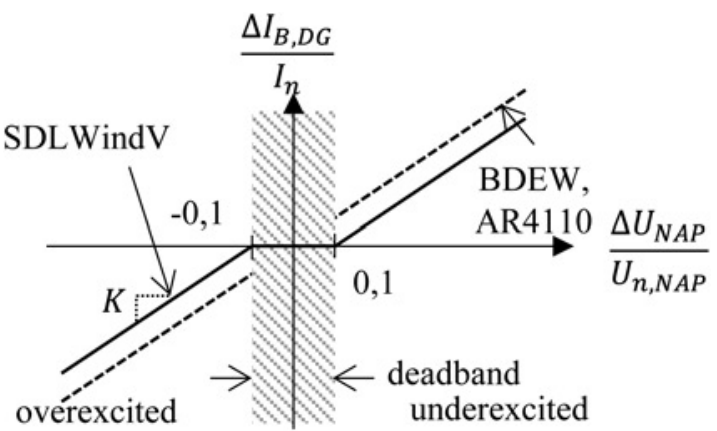

Fig. 3 Required additional steady-state DG reactive current injection for positive sequence voltage drops at the GCP

Depending on the voltage drop level the DG should inject an additional reactive current according to the characteristic line shown in Fig. 3. Here the K-factor describes the slope of the line, which is chosen by the grid operator between 2 and 10 .

There is no specification for the active current injection of the DG. However, for PF the current amount is limited to a value close above the rated current due to the PF valve rating. This can be realised by the computing-based injection control. For DFIG an additional short-current input driven by the rotating machine to be expected. For this reason, a current up to $6 I_{\mathrm{n}}$ during the dynamic settling process is possible [6]. The resulting dynamic behaviour for faults with high-voltage drop at the GCP is shown in Fig. 4.

Regarding the standardised requirements the dynamic settling should happen within $60 \mathrm{~ms}$ [5]. The behaviour is verified in the course of the plant and unit certification [7].

3.1.2 Measured DG behavior during dynamic grid support: To investigate the actual behaviour of commercially available PV inverters, measurements have been conducted in the testing centre of the Institute for High Voltage Engineering of the RWTH Aachen. The behaviour of four inverters of different manufactures with powers between 9 and $30 \mathrm{kWp}$ is analysed regarding their active and reactive currents injection during different symmetric and asymmetric grid faults [8]. The results show various diverse behaviour regarding the infeed during dynamic grid support. While the reactive current infeed copes with requirements, the active current infeed varies between zero and the maximum active current allowed by the total current limits in the steady fault state.

Furthermore, the settling times of the reactive and active currents after the fault occurrence are investigated (Fig. 5). The settling times of the reactive current appear to be lower than the required $60 \mathrm{~ms}$ for most fault cases. In contrast, the settling times of the active currents, which are not restricted in the current regulations, reach values up to $200 \mathrm{~ms}$. This can lead to problems, when low protection delays are necessary, since the infeed-currents within the transition time are

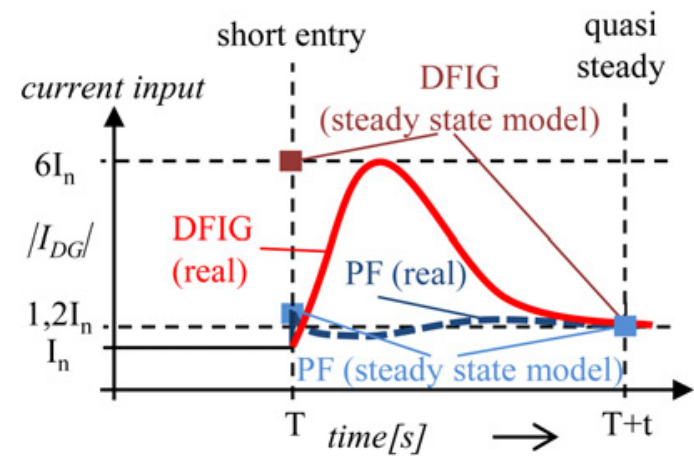

Fig. 4 Real DG-type dependent course of the current injection at the GCP after voltage drops and models 


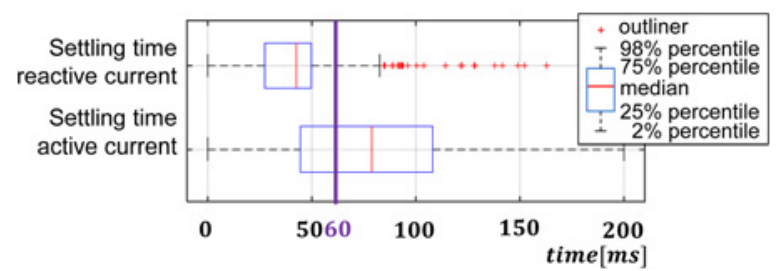

Fig. 5 Measured settling times of PV inverters with dynamic grid support for various symmetric and asymmetric faults

hardly predictable. Therefore future requirements should state restrictions for both the reactive and active current settling times.

\subsection{Impact on the network protection}

The described DG behaviour at the respective GCP has a corresponding impact on the network protection regarding Fig. 1. Three aspects are relevant:

(a) The reactive and active power infeed of the DGs downstream of a regarded protection devices aggregates at its installation point. Therefore the contribution of upstream network to the short-circuit current can be temporarily reduced (blinding).

(b) DGs in parallel feeders inject an additional short-circuit current amount, which is also registered by the MP at this feeder (OCP2 in Fig. 1). This effect can lead to erroneous activation of this device (sympathetic tripping).

(c) The mutual impact of the DG can extend the settling process significantly. This can lead to selectivity problems of the BP.

As described the network protection is parameterised for a tripping in $100 \mathrm{~ms}$ after the fault inception. As a result, there is an overlap between the DG-settling process and protection tripping.

In the following example, the settling process of all DG in Fig. 1 is simulated after a three-phase fault at the end of feeder 1. Fig. 6 shows the resulting phase current, which is relevant for the OPC0 pickup criteria.

In general, OPC 0 should ensure the $\mathrm{BP}$ within a tripping time after $0.4 \mathrm{~s}$ in the case of OCP1 fails. However, following issues are identified:

I. A delayed pickup due to a reduced initial short-circuit current at the protection device.

II. A hyperfunction due to a temporary leap into the quick time level. The relapse proceeds only after the shortfall below $I_{\mathrm{do}, \gg}$

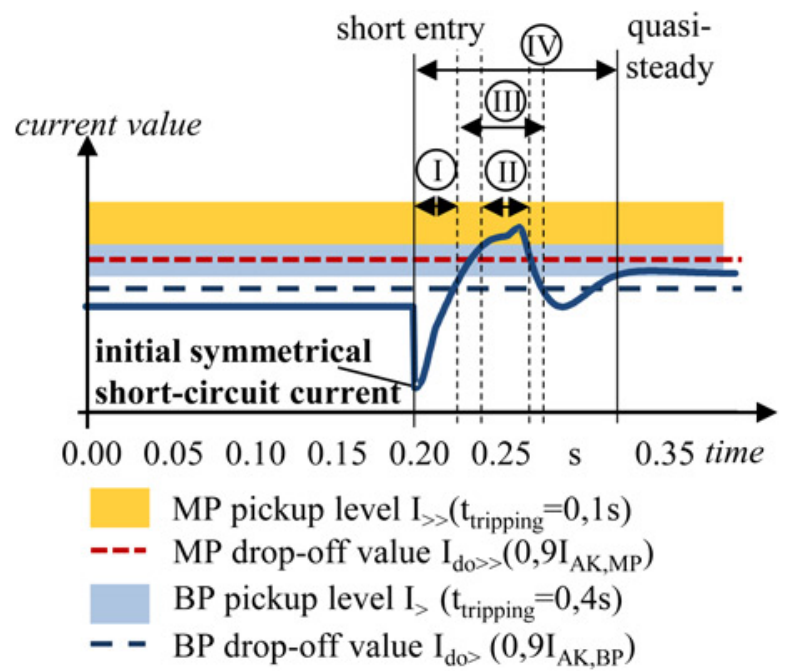

Fig. 6 Illustrative current course at OCP0 after fault occurrence

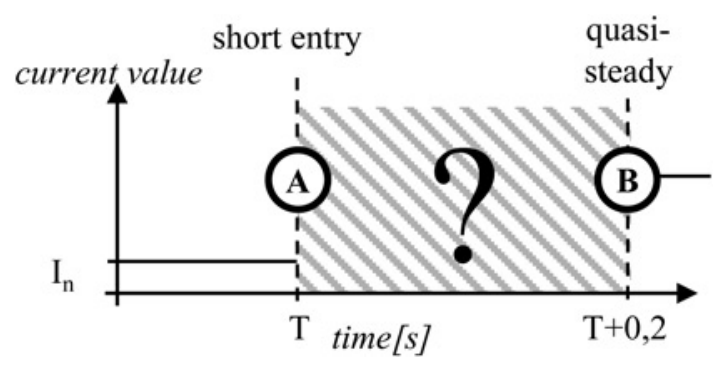

Fig. 7 Possible steady-state calculation points

III. Only a brief stay in the correct backup level, then temporary shortfall below $I_{>}$. due to the mutual influence of the DG. IV. Delayed permanent protective pickup and tripping in the backup level (pickup swing).

\subsection{New requirements for steady-state calculation methods}

The analysis of Fig. 6 illustrates, that a simplified steady-state calculation is not sufficient anymore for all protection designs in MV grids, even when the revised IEC 60909 is used [1]. A Rather at least two conditions have to be taken into account, as Fig. 7 illustrates:

(A) short-circuit failure entry,

(B) quasi-steady condition with settled DG current injection.

(A) Short-circuit failure entry can be estimated either by means of the revised standard calculation or it can be determined by the so-called exact calculation methods. (B) Quasi-steady condition with settled DG current injection therefore has to be calculated by implemented methods [9]. The modelled DG-behaviour is illustrated in Fig. 4. Not yet considered is the range in between, where the described settling effects occur.

\section{Modelling and simulation of the settling process}

\subsection{Network and DG models}

In order to evaluate the effect of the settling process on protection tripping numerous dynamic simulations have been undertaken, applying present approved models for network components and DGs (Fig. 4). Other dynamic effects are neglected.

The regarded $20-\mathrm{kV}$-grid has the topology shown in Fig. 1. Depending on the case at issues (a)-(c) specific worst-case DG-scenarios are considered a priori. Therefore as described above exclusively DFIGs are used.

\subsection{Measurement value evaluation of protection devices}

In the simulations root mean squares (RMS) of the phase currents are determined. There is no detailed modelling used for the measurement value the evaluation of OCPs. Rather it is assumed that common protective computing algorithm leads to a smooth measurement value [10].

\subsection{Simulation of the considered situations}

In general, the issued fault cases are two- and three-phase short-circuits in resonant-earthed networks and those with isolated neutral points at the end of feeder 1 (Fig. 1), which are reviewed in this example. Additional single-phased shorts are relevant at other neutral point treatment. However, further simulations have 
shown similar results in these cases as in the shown ones. By means of sensitivity analyses the influence of the DG behaviour is identified in the described cases at issues (a)-(c):

(a) 'Blinding' of the MP: Relevant for consideration is an assumed high installed power of DFIGs in feeder 1 in Fig. 1. Therefore the inertial short current leads to a reduced current at the protection device and a delayed pickup $(I)$ is thinkable.

(b) 'Sympathetic tripping' of the parallel MP: Relevant for consideration is an assumed high installed power of DFIGs in feeder 2 according to Fig. 1. Therefore the initial short current at OPC2 increases and a hyperfunction of this device is thinkable, either dynamic according to issue II or even steady state.

(c) Selectivity problems of the BP: Relevant for consideration is an assumed high installed power of DFIGs in feeders 1 and 2 according to Fig. 1. Therefore, a malfunction of OPC0 is thinkable, again both due to dynamic issues III and IV as well as steady state.

\section{Detected protective malfunctions}

Selected results of the described dynamic simulations are shown in Fig. 8 .

Based on these results we come to the following conclusions.

(a) 'Blinding' of the MP: In case (a) the maximal phase current measured at OCP1 $\left(I_{K 2, P h 1}^{(\mathrm{OCP} 1)}\right)$ can be seen to directly reach the quick time level. The currents in the other phases are also influenced significantly. Although the current is reduced slightly the protection stimulus remains. The whole time the correct tripping after $100 \mathrm{~ms}$ is expected. Therefore, a blinding due to dynamic effects does not occur.

(b) 'Sympathetic tripping' of the parallel MP: In case (b) the corresponding measured current at OCP1 leads to an undelayed pickup of this protection device. However, OCP2 is stimulated in the fast time stage simultaneous. Whereas the current over OCP1

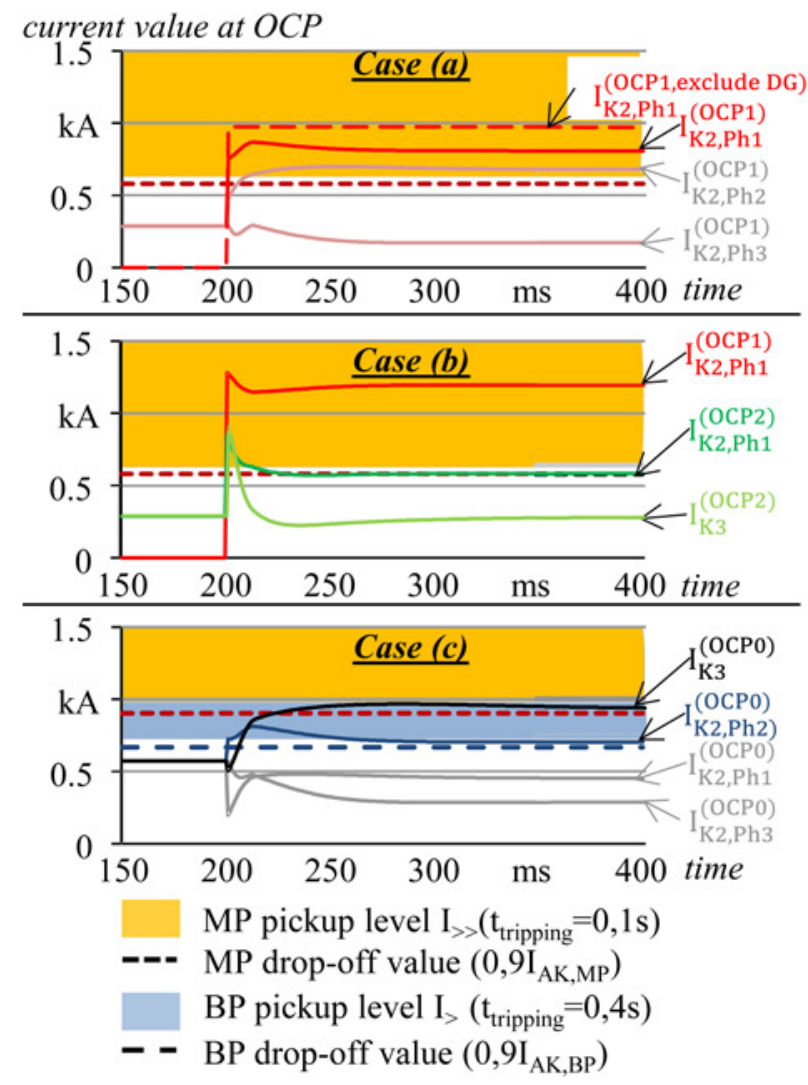

Fig. 8 Dynamic simulation of the current course at the PCP in (a)-(c) remains in the same level, the value at OCP2 falls back below the pickup level, but in a two-phased fault only close to the range of the drop-off value. Therefore, there is a threat for unselective tripping of OPC2 after $100 \mathrm{~ms}$.

(c) Selectivity problems of the BP: In a two-phase fault tripping on $I>$, OCP2 is delayed. The phase current is reduced after a view milliseconds but remains higher than the drop-off value $I_{>\text {,do }}$.

Regarding real OCP algorithms it is not certain that the necessary pickup will take place. However, with the aim of the described steady-state calculation methods this evaluation can be reached sufficient.

In addition, with regards the three-phase fault current there is a potential unselective pickup in the quick time level. However, this problem can be avoided with the use of pickup interlock parameterisation because of the pickup in quick time level of OCP1.

\section{Derived rules for the evaluation and prevention of protective functions using steady-state approaches}

The protection parameterisation can be approximately evaluated by the calculation of the conditions in the fault inception (A) and in the settled state (B). The example shown dynamic simulations for (a)-(c) shown in Fig. 8 permit an evaluation of the protective issues I-IV pictured in Fig. 6.

I. It is identified a $20 \mathrm{~ms}$ pickup delay by the use of the recommended relay separation of $300 \mathrm{~ms}$ no protective issue are to be expected.

II. A hyperfunction seems to be possible. All error types should be considered. In addition, the mentioned pickup security factor should be maintained reducing this risk.

III. A fall back out of the pickup level while a fault case might be possible. Therefore considering the drop-off value of the OCP is necessary by calculating (B), if a pickup is identified in (A) by the use of in Fig. 4 shown model assumptions.

IV. A pickup swing has not been identified in the regarded grids.

\section{Summary}

In distribution grids with a high amount of installed DG remaining in operation during a fault case and participating at a dynamic grid support by fast fault currents, used steady-state calculation methods have to be reconsidered. Besides the determined inertial short-circuit current the state with settled DG currents have to be taken into account.

Furthermore, there are additional dynamic effects in high DFIG-penetrated grids, which could influence the protective behaviour. The real settling times have been analysed in laboratory. The resulting durations lay in between 60 and $200 \mathrm{~ms}$ for PF. For DFIGS similar ranges are expected.

The influences of the DG settling on the grid protection have been investigated in dynamic simulations by means of wort-case-assumptions for issues (a)-(c). For a priori identified resulting dynamical challenges I-IV rules have been derived for the protection design. The risk of unintended behaviour of the grid protection can be minimised by the use of these general assumptions. Additional guidelines for the parameterisation of grid protection to avoid such problems have been given.

Consequently, a further use of steady-state calculation methods is possible with consideration of the fault inception (A) and the steady state after DG settling.

\section{References}

Kurzschlussströme in Drehstromnetzen: 'DIN EN 60909-0 (VDE 0102)', 2016 FNN: 'Leitfaden zum Einsatz von Schutzsystemen in elektrischen Netzen', 2009

3 VDN: 'TransmissionCode 2007: Netz- und Systemregeln der deutschen Übertragungsnetzbetreiber', 2007 
4 bdew: 'Erzeugungsanlagen am Mittelspannungsnetz: Richtlinie für Anschluss und Parallelbetrieb von Erzeugungsanlagen am Mittelspannungsnetz', Berlin, 2008

5 Verordnung zu Systemdienstleistungen durch Windenergieanlagen: SDLWindV, 2009

6 VDE-AR-N 4110: 'Erzeugungsanlagen am Mittelspannungsnetz: Technische Mindestanforderungen für Anschluss und Parallelbetrieb von Erzeugungsanlagen am Mittelspannungsnetz', VDE, Berlin, 2016

7 FGW: 'Technische Richtlinien für Erzeugungsanlagen: Teil 3: Bestimmung der Elektrischen Eigenschaften von Erzeugungseinheiten und -anlagen am Mittel-, Hoch- und Höchstspannungsnetz', 2013
8 Glinka, F., Bertram, R., Wippenbeck, T., et al.: 'Protection of today's and future low voltage grids with high DG penetration: laboratory and simulative analysis of blinding of protection with inverters'. The 13th IET Int. Conf. Developments in Power System Protection (DPSP), 2016

9 Wippenbeck, T., Jäkel, M., et al.: 'Development and cross-validation of short-circuit calculation methods for distribution grids with high penetration of inverter-interfaced distributed generation'. 23rd Int. Conf. Electricity Distribution, 2015

10 Blackburn, J.L., Domin, T.J.: 'Protective relaying: principles and applications' (CRC Press, Boca Raton, 2007, 3rd edn.) 